\title{
Creando e-SPACIOS para la participación popular en el patrimonio: el caso de la comunidad gay en Sitges
}

\author{
Puigbò, J. Tardío, P. Ortega, H.- Universidad Autónoma de Barcelona \\ Proyecto de Investigación Lakatos en Antropología (PILA) ${ }^{1}$
}

DOI:http://dx.doi.org/10.5565/rev/periferia.585

\section{Resumen}

Este artículo presenta una propuesta para el tratamiento del patrimonio de forma horizontal e interactiva: el e-SPACIO, así como la prueba piloto que hemos realizado con la comunidad gay de Sitges. Hasta la fecha, hemos presenciado procesos de patrimonialización verticales y arbitrarios, dirigidos desde unas instituciones que funcionan como lobbies de la memoria. Este hecho ha provocado la exclusión de algunos sectores de la sociedad en la construcción de su pasado y, en consecuencia, en el entendimiento de su presente, condicionando a la vez su futuro. Nosotros proponemos el e-SPACIO para afrontar esta problemática. Se trata de una herramienta para la construcción patrimonial de modo horizontal, colectivo y que vive tanto en el espacio físico como en el telemático, haciendo uso de las TIC (tecnologías de la información y de la comunicación).

Palabras clave:Patrimonio, patrimonio LGTBI+, TIC, TIC aplicadas, nuevos modelos patrimoniales, gestión patrimonial, Sitges.

\begin{abstract}
This article tries to present our proposal for the treatment of heritage: the eSPACIO, and the test we have done with the gay community Sitges. Till the date, we have witnessed processes of vertical and arbitrary heritage building, guided from institutions then working as lobbies of memory. This has led to the exclusion of sectors of society in the construction of his past and, consequently, in the understanding of their present and their future uprising. We propose the e-SPACIO to address this problem. It is a tool oriented for processes of building heritage horizontally, collectively and living in both the physical space and telematics, by using the ICT (information and communication technology).
\end{abstract}

Keywords:Heritage, LGBTI+ heritage, ICT, ICT applied, new heritage models, heritage management, Sitges.

\section{Introducción: la problemática de la gestión patrimonial}

\footnotetext{
${ }^{1}$ Enviar correspondencia a: PILA (proyectolakatos@gmail.com), Josep Puigbò (josep.puigbo@e-campus.uab.cat), Pablo Tardio (pablo.tardio92@gmail.com), Héctor Ortega (ortegaflohec@gmail.com).
} 
Puigbo, J. Tardio, P, Ortega, H. (2016) Creando e-SPACIOS para la participación popular en el patrimonio: el caso de la comunidad gay en Sitges, perifèria $\mathrm{x}(\mathrm{y})$, Noviembre 2016

revistes.uab.cat/periferia

En este trabajo partimos de una concepción de patrimonio entendida como una construcción social e históricamente producida que alude a la cultura viva y cambiante producida por la sociedad. Así, aunque la concepción de patrimonio esté cargada con una connotación histórica (que deriva de la asunción del patrimonio como la herencia colectiva que recibimos de las generaciones que nos precedieron) debemos subrayar la esencia dinámica y sincrónica que subyace en este concepto (Arévalo 1996: 80).

En cierto sentido, el contenido del patrimonio cultural es el mismo que la antropología otorga a la cultura, es decir, las formas de vida sociales de los grupos humanos y sus manifestaciones, sean éstas materiales o inmateriales, pasadas 0 presentes (Arévalo 1996). De este modo, el terreno del patrimonio se nos presenta como un campo de aplicación de las técnicas y metodologías antropológicas.Igualmente, cuando hablamos de patrimonio, hay que tener presente que éste no es un concepto neutro sino que es utilizado ideológicamente. Por ello, no es de extrañar que durante años las instituciones gestoras del patrimonio -los lobbies de la memoria- representadas mayoritariamente por museos y otros agentes que gestionan la memoria histórica y las representaciones culturales, hayan tratado al patrimonio colectivo como un bien privado.

Estas instituciones patrimoniales juegan un papel decisivo en la gestión patrimonial, y desde los discursos oficiales se contempla y se refuerza su rol: "Ellas pueden conservar y reforzar la identidad social basada en una cultura monocromática y estrictamente nacional. $\mathrm{O}$, al contrario, pueden conducir a la creación de una identidad verdaderamente intercultural y a una comprensión más amplia del "nosotros" (MECD 2005)². Así, estas instituciones definen qué es patrimonio y qué no, imponiendo un pasado determinado al resto de la sociedad. Estas decisiones, tomadas por unos pocos han provocado procesos de patrimonialización elitistas e incluso paternalistas, rechazando activamente una visión integral y holística del patrimonio,obviando muchos de los aspectos no visibles e inmateriales de la cultura. De este modo, se ha tratado la memoria y el pasado de una comunidad

\footnotetext{
${ }^{2}$ Ministerio de Educación, Cultura y Deporte, Gobierno de España (2005).
} 
Puigbo, J. Tardio, P, Ortega, H. (2016) Creando e-SPACIOS para la participación popular en el patrimonio: el caso de la comunidad gay en Sitges, perifèria $\mathrm{x}(\mathrm{y})$, Noviembre 2016

revistes.uab.cat/periferia

como si no le pertenecieran a ésta.El resultado de esta gestión patrimonial unilateral es la exclusión de los sectores desfavorecidos de la sociedad en la construcción del patrimonio y, en consecuencia, el olvido cultural de determinadas comunidades inducido por procesos de filtraje patrimonial por parte de sectores hegemónicos. Es decir, la patrimonialización como mano derecha de una ideología desarrollada lejos del servicio común, y por tanto muy alejada de la noción de patrimonio cultural como generador de recursos que contribuyan al incremento de la calidad de vida de la población (Arévalo, 1996:81). Sin ser ingenuos, hay que comprender la fuerza política que se desata del patrimonio. Y su uso puede suscitar herramientas de control. En el sentido que -como ya se apuntaba en la novela 1984de Orwell- quién controla el pasado controla el futuro y quien controla el presente controla el pasado.

Un caso ilustrativo es el de la comunidad gay en Sitges, donde realizamos nuestra prueba piloto. La presencia de la comunidad gay ${ }^{3}$ en Sitges es innegable. Su presencia en las calles y en la vida social de la urbe transcurre de forma cotidiana, situando a la ciudad como una de las escenas gay más notables de Europa. Esta situación puede conducirnos a pensar que el patrimonio de esta comunidad (presente en el territorio desde mediados del siglo XIX) debe estar vigente en los centros patrimoniales, pero nada más lejos de la realidad. En Sitges -y con esto nos referimos a las elites encargadas de la administración del patrimonio local- se ha optado por un pasado más cómodo: el modernismo, con su máximo exponente en el pintor y escritor Santiago Rusiñol.

La relevancia que se da a este patrimonio, en oposición a otros, es un claro ejemplo de un procedimiento de filtraje involucrado en un proceso de patrimonialización arbitrario que ha provocado la invisibilización del patrimonio cultural de la comunidad gay masculina de Sitges.

Esta arbitrariedad se evidencia -irónicamente- enel posible vínculo existente y sospechosamente olvidado entre el movimiento modernista en Sitges y la

\footnotetext{
${ }^{3}$ Más adelante detallamos por qué nos referimos a gay y no a LGTBI+ (apartado 4).
} 
Puigbo, J. Tardio, P, Ortega, H. (2016) Creando e-SPACIOS para la participación popular en el patrimonio: el caso de la comunidad gay en Sitges, perifèria $\mathrm{x}(\mathrm{y})$, Noviembre 2016

revistes.uab.cat/periferia

comunidad gay de la misma ciudad. Ambos colectivos empiezan a aparecer en la ciudad a mediados del siglo XIX. Las familias burguesas de Barcelona enviaban a sus hijos homosexuales varones a la casa de campo de Sitges, dónde podíanllevar una vida lejos de los rumores de la aristocracia de la ciudad condal. De forma paralela, empieza a surgir un fuerte movimiento de artistas de vida bohemia en la ciudad. Estos dos fenómenos locales, en la actualidad, representan dos de los principales elementos turísticos de la ciudad.

Como hemos visto, ambos podrían mantener vínculos comunes, pero el proceso de patrimonialización llevado a cabo no solo no los relaciona, sino que obvia a uno de ellos, silenciando la construcción del pasado del colectivo gay en esta ciudad. Patrimonio que -gracias a iniciativas populares al margen de las instituciones- se rescata poco a poco del olvido.

\section{Referentes teóricos}

Como respuesta a este contexto de expropiación a manos de lobbies de la memoria, necesitamos construir una definición de patrimonio desde un marco teórico más democrático, mediante el cual desarrollar nuestra metodología de intervención patrimonial.Si contemplamos la evolución del concepto de patrimonio, podemos apreciar que se está experimentado un incremento crítico en las reflexiones antropológicas alrededor de esta noción. Estas nuevas apreciaciones del concepto de patrimonio en antropología suponen una revisión teórica y metodológica que pretenden superar planteamientos marcados por componentes coloniales, impropios del marco antropológico contemporáneo.

De este modo, vemos un giro etnográfico(Fernández de Rota, 2009) en la forma de entender el patrimonio, el cuál comprende este concepto desde una perspectiva sociocultural estableciendo valor en la contextualización de la vida social y comportamiento de los sujetos y de sus objetos materiales. En este sentido, se están revisando y examinando antiguas sugerencias que figuraban un patrimonio folklorista, ligado fuertemente a nacionalismos y regionalismos, que erigían una visión estereotipada de las comunidades, más propias del espíritu del pueblo (volkgeist) y de determinadas aspiraciones que de la realidad. 
Puigbo, J. Tardio, P, Ortega, H. (2016) Creando e-SPACIOS para la participación popular en el patrimonio: el caso de la comunidad gay en Sitges, perifèria $\mathrm{x}(\mathrm{y})$, Noviembre 2016

revistes.uab.cat/periferia

Las reorientaciones teórico-metodológicas que están aconteciendo pretenden recuperar el valor y los significados del patrimonio desde la pluralidad de la identidad sociocultural (Fernández de Rota, 2009: 157). Avanzando, de este modo, de un modelo patrimonial que homogeneizaba las identidades colectivas, derivando en una cosificación de objetos y costumbres propios de una museología estática, hacia una visión holística, dinámica y justa del patrimonio cultural.

Es en este contexto convulso y de re-significación del concepto de patrimonio donde emerge nuestra propuesta. Partiendo de Bortolotto (1996), comprendemos que el patrimonio es un elemento cargado de connotación histórica pero que no está atado al pasado y a la herencia colectiva, sino que resulta dinámico. Con esto, comprendemos que pese a las cargas históricas el patrimonio puede resultar anacrónico, en el sentido que una comunidad puede rescatar una tradición o uso social determinado que hace tiempo que no se desarrolla por motivos de identidad, políticos u otros.

En segundo lugar, encontramos en Prats (2005) la explicación al uso político del patrimonio. Como muestra el autor, considerar el patrimonio a la vez como un constructo social y una invención social no es un oxímoron, sino más bien un dualismo. Es decir, el patrimonio puede ser un elemento rastreable históricamente hasta su origen, o bien un conjunto de elementos y rasgos culturales que una comunidad decide alzar como patrimonio. En este caso, entendemos que los lobbies de la memoria han elegido unas características culturales determinadas con un uso político determinado. Ahora bien, este uso del patrimonio no tiene por qué ser negativo, siempre y cuando evidenciemos la finalidad y entendamos que el medio puede usarse también para el servicio común.

En tercer lugar, aplicamos el concepto de agencia de Gramsci (citado en Pizza, 2005) para ver en el patrimonio no solo un elemento decorativo y de presentación, tampoco de resistencia solamente, sino que habita en él una capacidad subversiva. Es decir, el patrimonio como agente transformador mediante el cual las comunidades implicadas, en otro tiempo excluidas y apartadas, pueden a través de 
Puigbo, J. Tardio, P, Ortega, H. (2016) Creando e-SPACIOS para la participación popular en el patrimonio: el caso de la comunidad gay en Sitges, perifèria $\mathrm{x}(\mathrm{y})$, Noviembre 2016

revistes.uab.cat/periferia

él modificar las condiciones de su presente.

Por último, entendemos que el concepto "cultural inmaterial" tiene cierta utilidad para la adecuada conceptualización del patrimonio. Somos conscientes de la problemática de este concepto y de su auge como concepción de moda en los últimos años, pero nos resulta útil como concepto bisagra y analítico, ya que a través de él podemos explicar desde un punto de vista antropológico todo aquel patrimonio que no es meramente material.

\section{Nuestra propuesta: el e-SPACIO}

En respuesta a la visión utilitarista y elitista del patrimonio que antes hemos expuesto, nace nuestra propuesta metodológica: el e-SPACIO. Huyendo de una perspectiva ingenua, contemplamos el patrimonio como una herramienta política que debe ser construida desde la comunidad a la cual pertenece, pero que debe basarse en la construcción de conocimiento científico mediante métodos etnográficos.

EI e-SPACIO comulga los espacios virtuales y los espacios físicos para generar sinergias entorno al patrimonio y los agentes implicados en él, primordialmente entre la comunidad a quién le pertenece. A través del uso de los hándicaps inherentes en los espacios telemáticos -una percepción del tiempo alterada, una relación social en un soporte condicionable y un difuminado de la delimitación de los espacios o fronteras (Faura, 1998)- comprendidos en el ciberespacio que ofrecen las TIC (principalmente las redes sociales, páginas web y aplicaciones móvil), pretendemos desarrollar un entorno adecuado para el diálogo patrimonial y su construcción dentro de la comunidad propietaria de su patrimonio. Asimismo, también es necesario idear los acuerdos espaciales en los e-SPACIOS. Es decir, tenemos en cuenta que en el ciberespacio existe la posibilidad de introducir sesgos por parte de las personas investigadoras ya que se manejan las relaciones sociales mediante una "lógica online" (Picciulo 1998), pero aprovechamos la artificialidad del contexto relacional para fomentar los elementos que se quieren potenciar: diálogo, participación, horizontalidad y reciprocidad, entre otros. 
Puigbo, J. Tardio, P, Ortega, H. (2016) Creando e-SPACIOS para la participación popular en el patrimonio: el caso de la comunidad gay en Sitges, perifèria $\mathrm{x}(\mathrm{y})$, Noviembre 2016

revistes.uab.cat/periferia

De este modo, el e-SPACIO fomenta las sinergias entre las redes comunitarias implicadas en un patrimonio específico incorporando a los espacios físicos y en las lógicas inmateriales -ambas partes constitutivas del patrimonio- el ciberespacio: el lugar telemático donde tratar este patrimonio. Fortaleciendo y poniendo en común los diversos sectores de una comunidad determinada, llamadas al diálogo y a la construcción de su patrimonio, evitando la jerarquización de este proceso patrimonial como sucede con los modelos clásicos. A menudo más pensados en la conservación del patrimonio que en el uso y su construcción desde la propia comunidad. Aunque no somos contrarios a la conservación cultural, a menudo vemos en esta práctica una custodia que despoja de las comunidades la opción de participar de su herencia. En este sentido, defendemos una reorientación de la conservación del patrimonio ya señalada por Hufford (1994: 4): "el conservacionista cultural puedeaplicar su investigación al servicio de grupos que no pertenecen a la cultura dominante, con el fin de formar y sostener una sociedad culturalmente pluralista"4

Entendemos que la exposición del concepto de modo teórico puede resultar confuso -por eso más adelante expondremos su funcionalidad práctica mediante el caso específico del patrimonio de la comunidad gay masculina en Sitges- pero en este punto es necesario detallarlo.

\section{Etapas del proyecto y del e-SPACIO: modelo PILA de tratamiento patrimonial}

Para desarrollar nuestro modelo de tratamiento patrimonial hemos partido del modelo propuesto por Becerra en 1999. Vemos, en su modelo, un buen inicio para la gestión participativa del patrimonio cultural. Aunque debe matizarse y reinventarse para conseguir nuestros objetivos.

Así, la primera incursión que propone Becerra (1999) en su modelo es idéntica a nuestra fase: la investigación. A su vez, forma parte del punto de partida de nuestro modelo: la "Fase Etnográfica". Esta parte consta del riguroso trabajo de

\footnotetext{
${ }^{4}$ Traducción propia.
} 
Puigbo, J. Tardio, P, Ortega, H. (2016) Creando e-SPACIOS para la participación popular en el patrimonio: el caso de la comunidad gay en Sitges, perifèria $\mathrm{x}(\mathrm{y})$, Noviembre 2016

revistes.uab.cat/periferia

campo antropológico para estudiar, delimitar, comprender y entender el patrimonio de una comunidad. A continuación, Becerra propone la conservación del patrimonio, mientras que para nuestro modelo esto supone un salto abrupto debido a que nosotros estamos aún en una fase de apertura etnográfica. Esto es el primer paso de la segunda parte de nuestro modelo: la "Fase Postetnográfica". Es decir, proponemos "Abrir la etnografía" para llevar a la comunidad los resultados etnográficos que les permitan una visión antropológica del patrimonio. Siguiendo, Becerra plantea a continuación difundir ya el patrimonio, esto supone de nuevo una divergencia y ruptura entre ambos modelos, pues nosotros aún nos vemos incapacitados para conservar un patrimonio que, por otro lado, está en construcción. Nuestro modelo al "Abrir la Etnografía" propone una construcción del patrimonio de forma colectiva esto creemos que se consigue a través del diálogo horizontal con los agentes de la comunidad y mediante las TIC. Haciendo uso en esta parte de las herramientas TIC elegidas (web, aplicación móvil u otros), tanto para presentar los resultados de la etnografía de forma atractiva e interesante para las personas informantes -debemos ser conscientes que no para todo el mundo es atrayente una monografía- como para elaborar un diálogo entre grupo investigador-colectivo investigado y favoreciendo sinergias entre la propia comunidad.

Por último, Becerra (1999) propone una fase de "Restitución", que suponga una restauración y retorno de las alteraciones y molestias surgidas por la investigación, así como la vuelta a la comunidad de lo que es suyo: el patrimonio. Aquí existe una reconexión del enlace entre arquetipos, puesto que nuestro modelo apuesta también por la restitución a la comunidad. Ahora bien, mientras Becerra concibe el retorno en una fase final, el e-SPACIO propone una restitución transversal desde la segunda fase: la apertura de la etnografía. Asimismo, nuestra fase final se centra en la "Aplicabilidad práctica del patrimonio para la comunidad". Como hemos dejado en constancia, vemos un uso poliédrico del patrimonio, con lo cual entendemos que el grupo investigador puede deducir de la primera fase etnográfica -o a posteriori- las necesidades que hayan dejado latentes las personas que han 
Puigbo, J. Tardio, P, Ortega, H. (2016) Creando e-SPACIOS para la participación popular en el patrimonio: el caso de la comunidad gay en Sitges, perifèria $\mathrm{x}(\mathrm{y})$, Noviembre 2016

revistes.uab.cat/periferia

participado como informantes en el proceso de investigación. Creemos que es deber del equipo de investigación recogerlas para posteriormente presentarlas a la comunidad junto con propuestas para su resolución.En el caso de Gay Sitges Link el uso que debe tener el patrimonio -respondiendo a las necesidades expresadas por el colectivo- es el de la visibilización de la comunidad gay de Sitges. Esta visibilización debe centrarse tanto en la comunidad como en su patrimonio y debe ofrecer instrumentos para la lucha contra los procesos de estigmatización y la homofobia.

Comparativamente, la lógica de confrontación entre ambos modelos es la siguiente:

\section{TRATAMIENTO DEL PATRIMONIO}

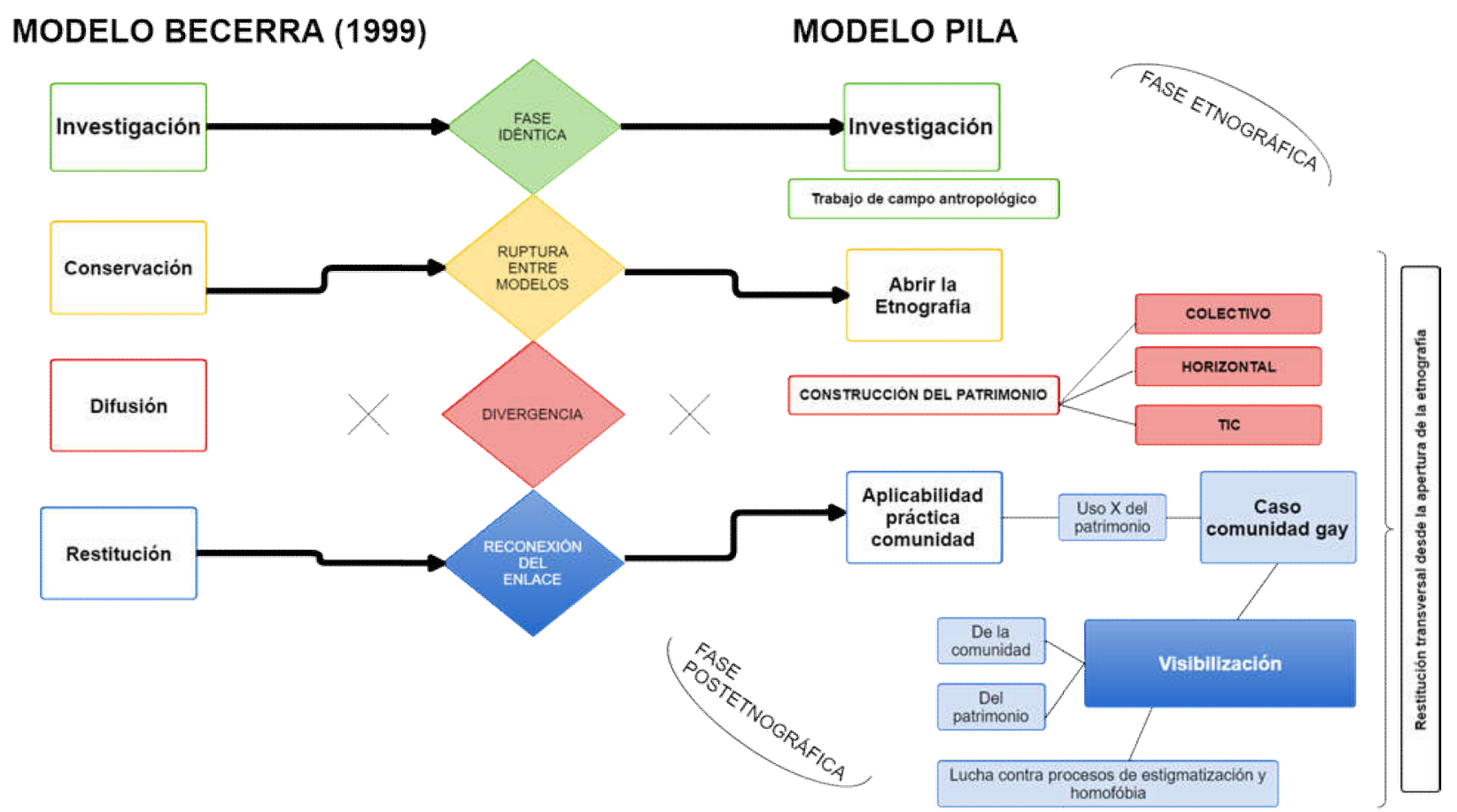

Ilustración I. Confrontación entre el modelo de tratamiento patrimonial de Becerra (1999) y PILA (2016).

Utilidades del e-SPACIO 
Puigbo, J. Tardio, P, Ortega, H. (2016) Creando e-SPACIOS para la participación popular en el patrimonio: el caso de la comunidad gay en Sitges, perifèria $\mathrm{x}(\mathrm{y})$, Noviembre 2016

revistes.uab.cat/periferia

Si nos centramos en las principales aportaciones y ventajas que emergen del eSPACIO nos encontramos, de forma preliminar, con tres puntos esenciales:

1) La posibilidad de mezclar un prisma emic y etic, a través de una perspectiva post-etnográfica. El término post no es un anhelo postmoderno, se trata simplemente de una conyugación descriptiva que remite a un proceso secuencial, como más adelante explicitaremos.

2) La construcción del patrimonio de forma horizontal y colectiva por parte de la comunidad.

3) Un altavoz entre la comunidad y la sociedad (a través del retorno y restitución del trabajo del equipo de investigación que ofrece herramientas a la comunidad que pueden ser útiles para diferentes fines).

\section{Limitaciones del e-SPACIO}

Siendo realistas y alejándonos de una perspectiva bienintencionada, divisamos en nuestro instrumento ciertas consideraciones a tener en cuenta. En primer lugar, el uso del e-SPACIO queda condicionado a comunidades que emplean cotidianamente o de forma regular las TIC (tecnologías de la información y de la comunicación). No sería lógica su aplicación en sociedades que no usen de forma frecuente estas tecnologías. No queremos, con esto, jerarquizar el concepto -ya que trabajamos en la posibilidad de aplicar otro formato de tratamiento del patrimonio para comunidades con otras características- simplemente supone la constitución de un modelo de tratamiento patrimonial pensado para unos contextos determinados.

$Y$, en segundo lugar, al traspasar las relaciones sociales a una plataforma (es decir, mediante un software) estamos condicionándolas. De todos modos, aunque el soporte lógico racional (Sarraméa 1999) que inserte el equipo de investigación (red social, página web o aplicación móvil) puede condicionar la interacción de los sujetos que se relacionen mediante ella, se intentará dirimir esta intrusión mediante la minimización de la intromisión para permitir que la relación social brote hasta su aparente naturalidad irracional. Además, se tendrá que tener en cuenta que esta imposición de una lógica online deja atrás premisas de la realidad social 
Puigbo, J. Tardio, P, Ortega, H. (2016) Creando e-SPACIOS para la participación popular en el patrimonio: el caso de la comunidad gay en Sitges, perifèria $\mathrm{x}(\mathrm{y})$, Noviembre 2016

revistes.uab.cat/periferia

convencional a contemplar para su análisis.

\section{Aplicación del concepto: prueba piloto}

Para comprobar la funcionalidad del concepto hemos llevado a cabo una prueba piloto. Debido a la falta de recursos nos hemos visto obligados a realizar el tanteo a pequeña escala. Por ello, nos hemos centrado exclusivamente en una sola entidad gay de la comunidad de Sitges: Gay Sitges Link. Se trata de una asociación sin ánimo de lucro con el objetivo de cohesionar socialmente y asistir a la comunidad gay y gay-friendly de Sitges -ya sean residentes o visitantes- mediante diferentes eventos y proyectos desarrollados y coordinados exclusivamente por voluntarios. Las características de la entidad han permitido que el proyecto sea coherente con sus principios, su lema resulta un puente entre nuestro modelo patrimonial y su idiosincrasia: "el orgullo de crear comunidad".

Como hemos comentado anteriormente, nuestra perspectiva se ve limitada al ámbito gay y no LGTBI+ como sería más enriquecedor, esto ha sucedido primordialmente porqué Gay Sitges Link, como indica su nombre, es una entidad centrada principalmente -aunque no totalmente- en el colectivo homosexual masculino. Así, al ver restringido el colectivo de aplicación, también hemos tenido que limitar nuestra mirada.

Las fases del proyecto han supuesto en sí mismas la aplicación de nuestro modelo de tratamiento del patrimonio: el e-SPACIO. Iniciamos nuestras tabulaciones teóricas con el modelo de Becerra (1999), el cual remite a cuatro fases principales en el tratamiento patrimonial: 1) Investigación, 2) Conservación, 3) Difusión y 4) Restitución. Si bien Becerra nos ofrece un buen punto de partida, encontramos que su modelo no presenta las particularidades idóneas para nuestro cometido. Debido a esto, nos vimos obligados a desarrollar nuestro propio modelo de tratamiento partiendo de los pilares que aposenta Becerra en su modelo de 1999.

\section{Expectativa teórica contra la realidad práctica}

Debido, nuevamente, a la escasez y falta de recursos no hemos podido abarcar todas las fases de nuestro proyecto. Aun así, hemos podido comprobar el 
Puigbo, J. Tardio, P, Ortega, H. (2016) Creando e-SPACIOS para la participación popular en el patrimonio: el caso de la comunidad gay en Sitges, perifèria $\mathrm{x}(\mathrm{y})$, Noviembre 2016

revistes.uab.cat/periferia

funcionamiento de diferentes fases del modelo. Las fases llevadas a cabo han sido: a) la aproximación etnográfica y un primario trabajo de campo; b) elaboración de resultados parciales; c) inicio de apertura de la etnografía y c) diseño de la aplicación móvil donde habita el e-SPACIO.

Por otro lado, no hemos podido poner en manos de la comunidad el e-SPACIO, hecho que no nos ha permitido elaborar unas conclusiones definitorias ni el inicio del proceso de patrimonialización en su última fase.

De todos modos, la incursión inicial al campo nos permite extraer algunas conclusiones relevantes para una futura aplicación del proyecto en su totalidad.

\section{Resultados de la fase etnográfica}

En primer lugar, era necesaria una aproximación sobre el uso de las nuevas tecnologías -concretamente de las redes sociales y hardware- para comprender si era adecuado implicar tecnologías de la comunicación en el proceso de patrimonialización. Por ello, decidimos que un cuestionario podría darnos información sobre la adecuación de la aplicación de las TIC.

La muestra del cuestionario se compone de 46 hombres homosexuales, con una media de edad de 36,2 años, que viven o visitan con regularidad en Sitges. Hay que tener en cuenta que para una futura aplicación del proyecto esta muestra deberá ser amplificada sustancialmente, consideramos que una muestra inicial de 300 sujetos seria adecuada.

Esta aproximación cuantitativa nos permite afirmar de forma preliminar que el colectivo homosexual de Sitges ve en las redes sociales de internet un buen medio para tratar problemas políticos y patrimoniales. Utilizando la escala de Likert, vemos que los encuestados opinan afirmativamente a que "las redes sociales en Internet pueden hacer que la gente se interese por los asuntos políticos" (con un promedio de 7.94 sobre 9). Por otro lado, también se observa que los informantes creen que "las redes sociales en Internet son una forma moderna de mantenerse al tanto de asuntos políticos" (con un promedio de 8.12 sobre 9).

Una vez constatado que ven en internet una plataforma útil de discusión y 
Puigbo, J. Tardio, P, Ortega, H. (2016) Creando e-SPACIOS para la participación popular en el patrimonio: el caso de la comunidad gay en Sitges, perifèria $\mathrm{x}(\mathrm{y})$, Noviembre 2016

revistes.uab.cat/periferia

comunicación política y patrimonial, es necesario analizar si existe un uso cuotidiano de las TIC.Si observamos los datos referentes a la familiarización del Smartphone, percibimos en consecuencia que el $96.88 \%$ de la muestra afirma tener un contacto familiar con el Smartphone. Por otra parte, esto solo responde a una aproximación subjetiva del individuo hacia el instrumento tecnológico, por eso son trascendentales los resultados sobre la frecuencia de uso.

En estos resultados observamos como la mayoría de encuestados utiliza el Smartphone entre 2 y 5 veces por hora (37.50\%). Por otro lado, el $56.26 \%$ usa el Smartphone más de 10 veces por hora, o entre 5 y 10 veces por hora, con el $28.13 \%$ de resultados respectivamente. Una muestra gráfica a continuación:

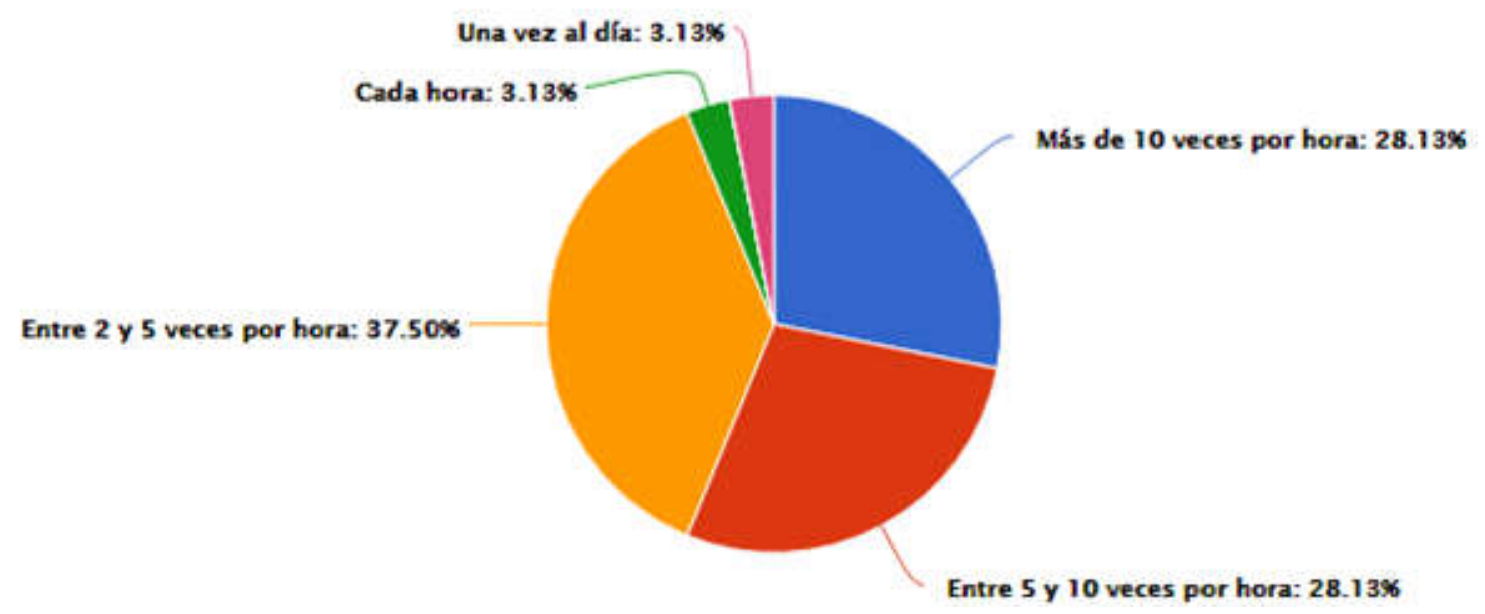

Ilustración II. Gráfico de frecuencia de uso del Smartphone.

Otro dato relevante, es que existe un acceso continuo a las redes sociales de internet por parte de los encuestados. En la pregunta centrada en los ingresos a redes sociales de internet en los últimos días, un significativo 93.75\% respondía que accedió en el último día, frente a un $6.25 \%$ que ingresó en los últimos siete días. 
Puigbo, J. Tardio, P, Ortega, H. (2016) Creando e-SPACIOS para la participación popular en el patrimonio: el caso de la comunidad gay en Sitges, perifèria $\mathrm{x}(\mathrm{y})$, Noviembre 2016

revistes.uab.cat/periferia

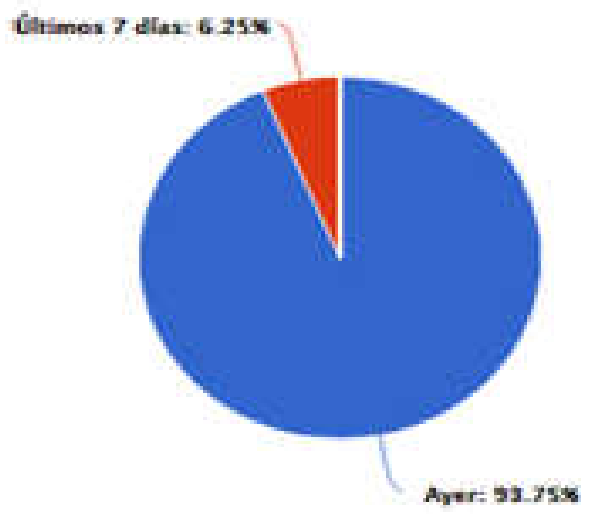

Ilustración III. Gráfico sobre el último acceso a redes sociales virtuales.

Estos datos ponen de manifiesto que internet, y las redes sociales en particular, son un espacio conocido y usado habitualmente por los encuestados. Del mismo modo, el Smartphone es un artilugio utilizado con regularidad y de forma diaria para la mayoría de encuestados. Una vez contrastado el uso de internet es necesario compartir los resultados de las entrevistas. Estas han sido diseñadas de modo semiestructurado y se han realizado con once socios de Gay Sitges Link.

Los resultados nos conducen a afirmar que existe una creencia mayoritaria de la existencia de un patrimonio gay en Sitges. Además, focalizan la localización de este patrimonio en comportamientos y lugares o espacios determinados, tales como bares, calles o playas determinadas. Esto, además, se ve de forma transversal en todos los coloquios:

"...las actitudes en la calle o en bares, un poco sabiendo a qué atenerse. Cuando estás en un bar gay si alguien te mira es porque está interesado, en un bar que no es gay o en la calle es distinto" Arnold (12/07/2016).

Del mismo modo, los informantes afirman -mayoritariamente- que al hablar de patrimonio es indispensable hacer mención de lugar formados por bares $y$ discotecas que en su momento fueron "historia y siempre siguen en la memoria". Además, estos espacios evolucionan mediante épocas y períodos según la opinión de los informantes. Con lo cual, observamos ciertos espacios 
Puigbo, J. Tardio, P, Ortega, H. (2016) Creando e-SPACIOS para la participación popular en el patrimonio: el caso de la comunidad gay en Sitges, perifèria $\mathrm{x}(\mathrm{y})$, Noviembre 2016

revistes.uab.cat/periferia

vinculados a unos comportamientos determinados que implican de forma emocional e histórica a los informantes.

Conclusivamente, podemos distinguir tres aspectos principalmente interesantes para este trabajo extraídos de la fase etnográfica: a) existencia de relaciones sociales dispares a las heteronormativas (sociabilidad), b) espacios físicos delimitados a la vida social gay (sociabilidad, interés sexual y comercios/instituciones) y c) uso habitual de las TIC, concretamente del Smartphone.

\section{Fase post-etnográfica}

A partir de la fase etnográfica obtenemos una aproximación al objeto de estudio que, aunque en el proyecto consumado resultaría el tránsito por un trabajo de campo completo, en esta aproximación piloto supone un primer contacto parcial para estudiar la viabilidad de la secuencia del e-SPACIO. Cabe destacar que expondremos solo el diseño de este período postetnográfico, puesto que las imposibilidades en relación a los recursos no nos han permitido elaborar una puesta en marcha desde la comunidad.

Esta etapa de nuestro modelo se centra en la apertura de los resultados de la comunidad, poniendo al alcance de su crítica y debate lo que el grupo de investigación ha conceptualizado como parte de su patrimonio. De este modo, la fase postetnográfica se configura y avala en los resultados etnográficos (lo que sería el resultado del proceso científico), pero también en la opinión y óptica de la colectividad (elementos que no tienen por qué responder a metodologías y usos científicos). Asimismo, queremos constatar que este período no responde ya a unas directrices propias del procedimiento científico, sino a las lógicas que sean aplicadas autónomamente por la comunidad respecto a su patrimonio. En este sentido, exponemos los resultados etnográficos delante de la comunidad, erigida en este momento como jueza y gestora de su herencia. 
Puigbo, J. Tardio, P, Ortega, H. (2016) Creando e-SPACIOS para la participación popular en el patrimonio: el caso de la comunidad gay en Sitges, perifèria $\mathrm{x}(\mathrm{y})$, Noviembre 2016

revistes.uab.cat/periferia

Para la construcción de este proceso comunicativo se han tenido en cuenta los resultados del trabajo de campo. Reconociendo que existe un elevado uso cuotidiano de las redes sociales de internet y del móvil inteligente, parece beneficiosa la creación de una plataforma basada en una lógica de red social -es decir, participación, interactividad, diálogo, elementos gráficos como fotografías, etc.- para smartphones. Esto es, en definitiva, una aplicación móvil.

El diseño del aplicativo móvil ha venido acompañado de un desarrollo escarpado, puesto que ha sido desarrollado por el equipo de investigación (perteneciente a las ciencias sociales) y no por expertos en telecomunicaciones. Esto pone de manifiesto la necesidad de equipos interdisciplinares también en el patrimonio. A pesar de las dificultades, diseñamos la aplicación mediante una organización basada en las siguientes premisas: facilidad de uso y acceso (gratuidad y sencillez).Dado que la facilidad de acceso era un pilar fundamental, la aplicación móvil ha de ser descargable gratuitamente desde los principales puntos de descarga de apps para Android y iOS. Por otro lado, siguiendo la simplicidad como objetivo, distinguimos en la aplicación los seis apartados siguientes:

1. Patrimonio. Apartado destinado a la exposición de forma atractiva para la persona participante de los resultados etnográficos. Además se destina un bloque de comentarios para que los y las participantes puedan comunicar su opinión respecto a la propuesta patrimonial y establecer un diálogo entre las diferentes intervenciones. A partir de la aproximación etnográfica, determinamos que uno de los centros de atención patrimonial deberían ser los espacios de sociabilidad gay de Sitges, concretamente los bares.

2. Gay Sitges Link. Sección centrada en la explicación de los labores de GSL y pensado, también, para el retorno a esta entidad.

3. e-SPACIO. Apartado donde se especifica el funcionamiento y bases del proyecto.

4. PILA - Proyecto de Investigación Lakatos en Antropología. Sección donde se 
Puigbo, J. Tardio, P, Ortega, H. (2016) Creando e-SPACIOS para la participación popular en el patrimonio: el caso de la comunidad gay en Sitges, perifèria $\mathrm{x}(\mathrm{y})$, Noviembre 2016

\section{revistes.uab.cat/periferia}

reúnen datos de contacto sobre el grupo de investigación.

5. Visibilización. Apartado ideado para el retorno a la comunidad. Se trata de unas adaptaciones propias de los cuadros del artista modernista Santiago Rusiñol mediante reivindicaciones LGTBI+.

6. $Q R$. Código de respuesta rápido creado para las futuras aplicaciones de la aplicación en el territorio. Por ejemplo: instalación de códigos QR en los diferentes lugares de sociabilidad gay en Sitges.
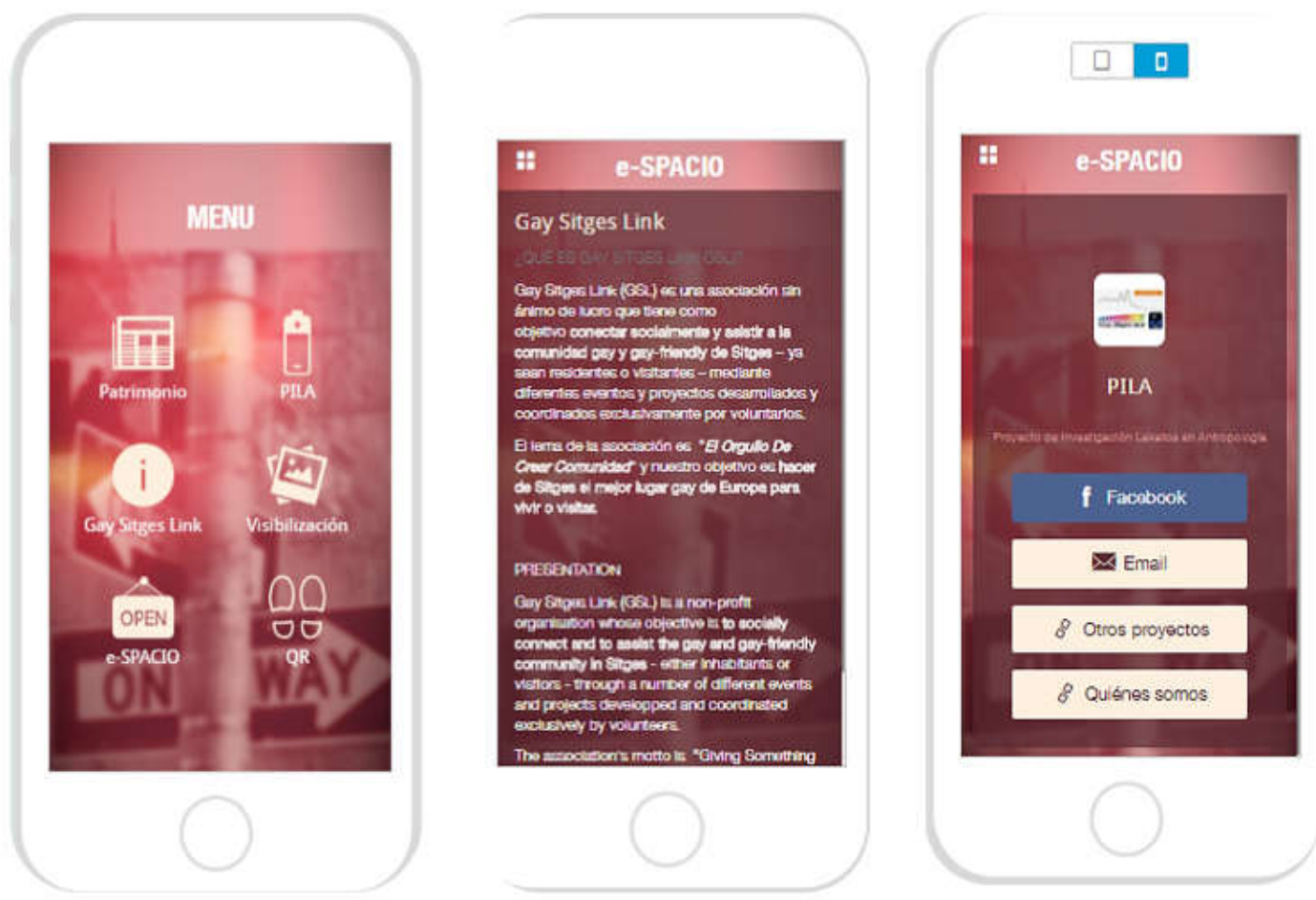

Ilustración V. Diseño aplicación móvil: Menú, Apartado Gay Sitges Link, Apartado PILA.

En la Ilustración $V$ se observa una muestra gráfica de la distribución de la aplicación móvil.

Respecto al punto más relevante, la sección sobre la presentación del patrimonio (con el título PATRIMONIO en la $A p p$ ), se ha estructurado mediante un planteamiento similar al de una red social: elementos gráficos, comentarios y texto y citas. De este modo creemos que es más colaborativo y accesible para el mayor 
Puigbo, J. Tardio, P, Ortega, H. (2016) Creando e-SPACIOS para la participación popular en el patrimonio: el caso de la comunidad gay en Sitges, perifèria $\mathrm{x}(\mathrm{y})$, Noviembre 2016

revistes.uab.cat/periferia

público posible. Hay que contemplar, para la aplicación completa del e-SPACIO en el marco de un proyecto total, la posibilidad de desarrollar otras herramientas interactivas, tales como audiovisuales y registros de voz. Eso, del mismo modo que una línea museográfica, debe procurar la correcta comprensión del discurso expuesto, a la vez que fomentar la participación.

En términos visuales, la sección sobre patrimonio centrado en los bares gays como espacios de sociabilidad particulares para la comunidad gay masculina de Sitges, se escenifica del siguiente modo:
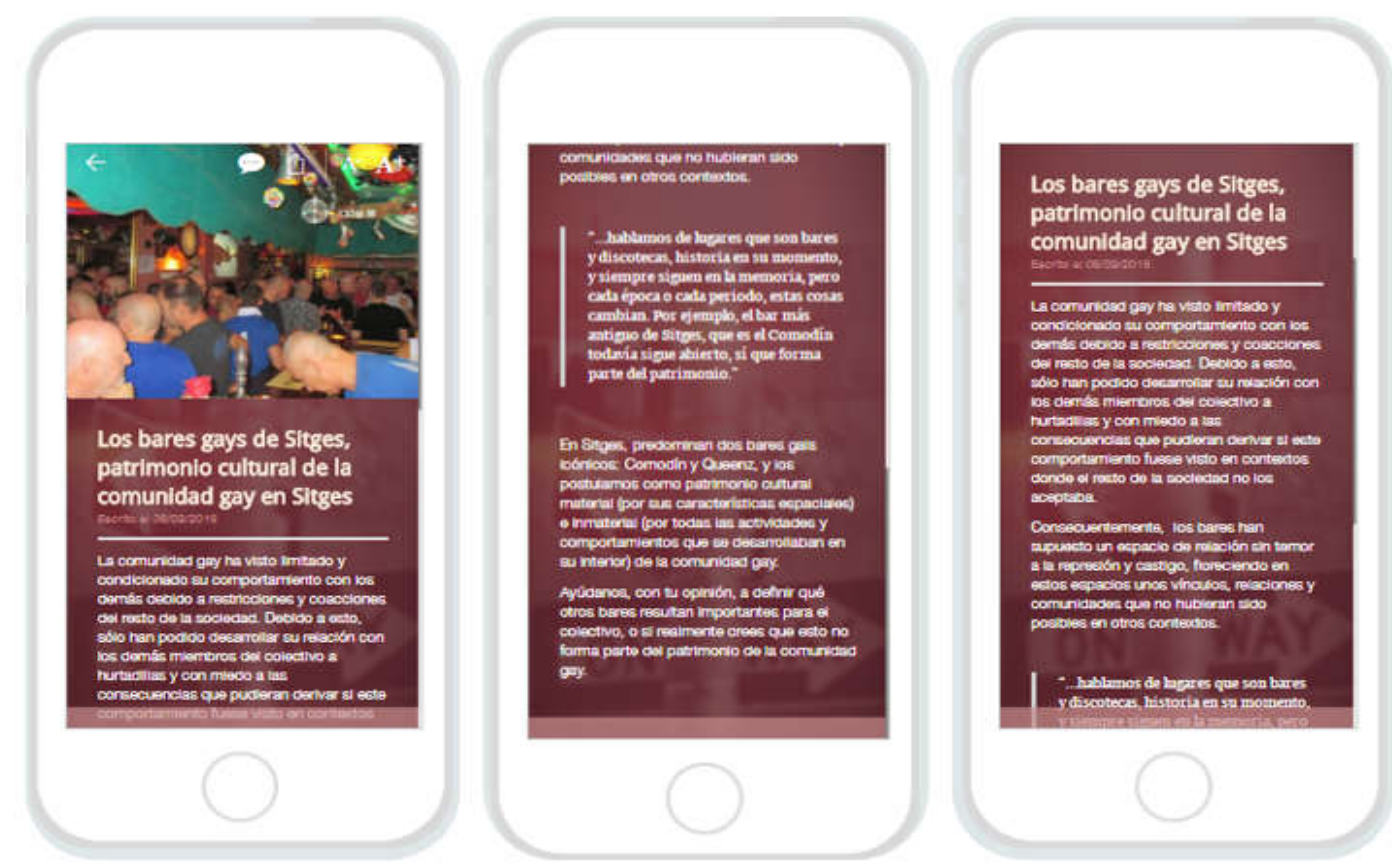

Para habilitar el diálogo por parte de la comunidad hemos establecido una

Ilustración VI. Diseño aplicación móvil: Apartado Patrimonio sección en este apartado centrado en recoger los diferentes comentarios y aportaciones.

A esta sección se accede desde el apartado de patrimonio y permite registrar para su posterior análisis los datos que la colectividad haga llegar a la plataforma. De este modo, se establece una comunicación entre el producto etnográfico y el 
Puigbo, J. Tardio, P, Ortega, H. (2016) Creando e-SPACIOS para la participación popular en el patrimonio: el caso de la comunidad gay en Sitges, perifèria $\mathrm{x}(\mathrm{y})$, Noviembre 2016

revistes.uab.cat/periferia

imaginario de los propietarios del patrimonio. Capacitando la perspectiva de la comunidad se evitan tratos paternalistas y verticales de su patrimonio, puesto que se reciben y escuchan las diversas opiniones.

A continuación una muestra gráfica:
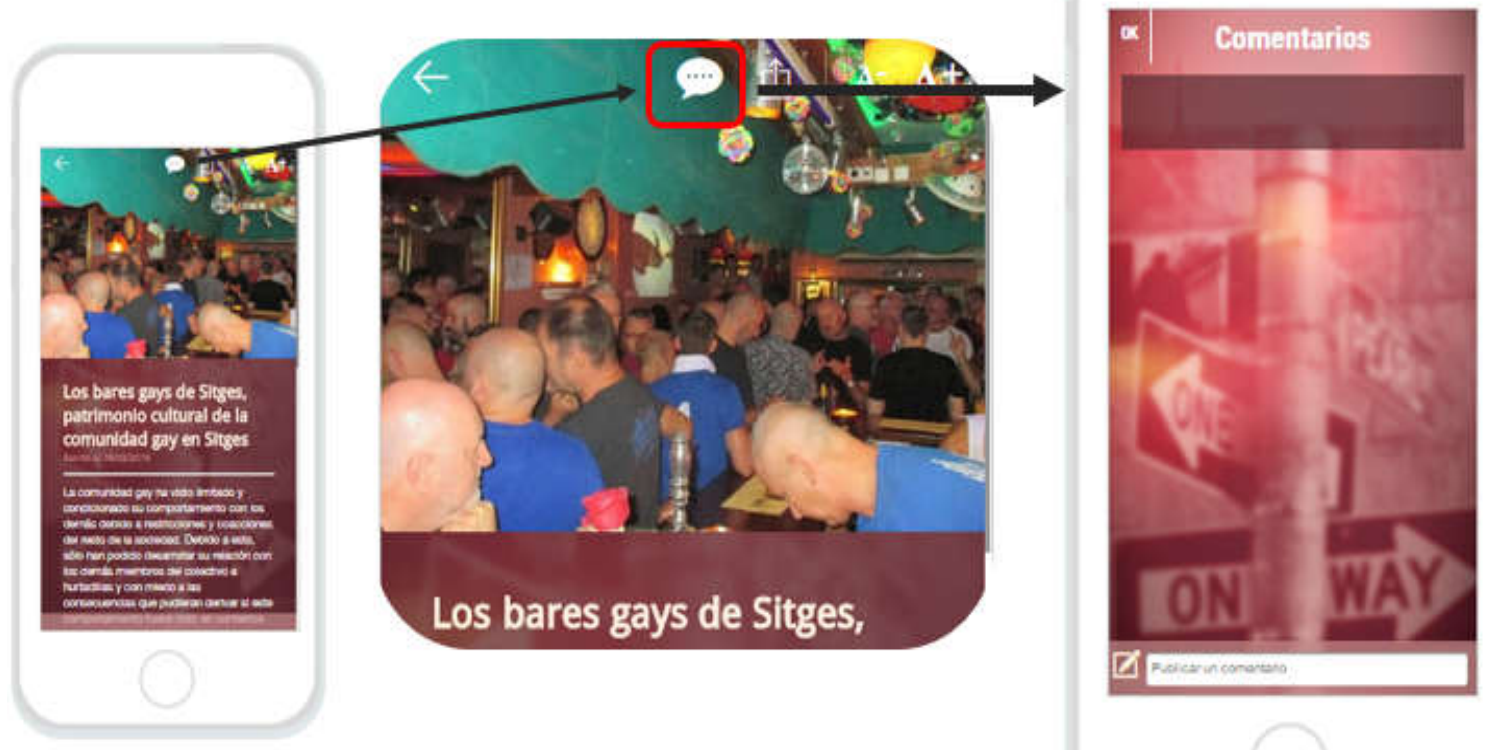

Ilustración VII. Diseño aplicación móvil: Apartado Patrimonio Comentarios.

\section{Consideraciones finales}

La gestión patrimonial actual se fundamenta en un procedimiento unidireccional y vertical que trata de modo paternalista a las comunidades y a su patrimonio. Nuestra propuesta no pretende ser una proposición dogmática, ni fomentar doctrina alguna, más bien se trata de una alternativa que surge en un contexto de 
Puigbo, J. Tardio, P, Ortega, H. (2016) Creando e-SPACIOS para la participación popular en el patrimonio: el caso de la comunidad gay en Sitges, perifèria $\mathrm{x}(\mathrm{y})$, Noviembre 2016

revistes.uab.cat/periferia

tendencias reivindicadoras de participación en los procesos sociales y a través de la colectividad en oposición a las lógicas existentes.

Frente al uso impositivo del patrimonio y su monopolio, se pretende fomentar la constitución de estructuras relacionales de forma horizontal en el tratamiento del patrimonio. Esto es, procurar una memoria colectiva que responda a los intereses de la comunidad. La elección fragmentaria del pasado es inherente en el proceso relacionado con la construcción conjunta de la identidad y el patrimonio, pero este desarrollo deben ejercerlo aquellas personas a las que pertenece; las verdaderas propietarias patrimoniales y que deben ser -por tanto- sus gestoras: la comunidad.

Asimismo, somos conscientes de que esta forma de tratamiento del patrimonio, pese a que pretende cuestionar la jerarquía entre instituciones gestoras y las comunidades, no alcanza a dilapidar las relaciones de poder que se establecen dentro de los grupos humanos (ya sea dentro de una sociedad o dentro de pequeñas comunidades). Es por esto por lo que, pese a que entendemos que no alcanza a eliminar las desigualdades en el proceso del tratamiento del patrimonio, creemos que puede ser una buena herramienta para fomentar el empoderamiento por parte de la comunidad de su patrimonio.

Por otro lado, en relación a su aplicabilidad, es pertinente considerar al e-SPACIO como un modelo de tratamiento patrimonial adaptable a comunidades $y$ poblaciones que no mantengan una alta frecuencia de uso de las tecnologías de la información y de la comunicación, puesto que las particularidades principales -y en consecuencia los objetivos primordiales- son la visión holística del patrimonio, el acercamiento antropológico y la participación horizontal de los agentes implicados. A su vez, el desarrollo mediante el uso de las nuevas tecnologías manifiesta elementos beneficiosos a tener en cuenta, como se ha evidenciado mediante el artículo: facilitación del diálogo y potenciación de la participación.

La prueba piloto evidencia que la secuencia modular de nuestra propuesta permite establecer una metodología de construcción de su patrimonio a la comunidad a 
Puigbo, J. Tardio, P, Ortega, H. (2016) Creando e-SPACIOS para la participación popular en el patrimonio: el caso de la comunidad gay en Sitges, perifèria $\mathrm{x}(\mathrm{y})$, Noviembre 2016

revistes.uab.cat/periferia

través de una investigación antropológica. La aproximación etnográfica permite desarrollar una fase postetnográfica que admite poner a debate la perspectiva del grupo investigador, desarticulando posiciones verticales. Ahora bien, debido a las limitaciones del proyecto no ha sido posible poder evaluar cómo se integra en la comunidad gay masculina de Sitges la aplicación móvil. Una vez más, topamos con que la situación contextual incapacita la puesta en práctica de propuestas alternativas al margen de las instituciones que monopolizan los recursos.

Por último, vemos en el patrimonio una oportunidad para transformar la calidad democrática de nuestras sociedades, puesto que es imposible formular una sociedad ecuánime desde un pasado impuesto. Por eso creemos que nuestra propuesta formula nuevas conjeturas que permitan tratar el patrimonio como legado y herencia pública y a sus comunidades con la suficiente madurez que les atañe. Así, descartaríamos el modelo de tratamiento patrimonial actual, formulado desde lobbiesde la memoria que despojan a los colectivos de su ayer y sus recuerdos.

\section{Bibliografía}

Arévalo, J.M. (1996). El patrimonio antropológico. A propósito de la realidad cultural Extremeña. Revista Murciana de Antropología, N03, 79-98.

Bortolotto, Ch. (2014). La problemática del patrimonio cultural inmaterial". Culturas. Revista de Gestión Cultural. Vol. 1, No1, 1-22.

http://dx.doi.org/10.4995/cs.2014.3162

Faura, R. (1998) "La cultura local en el ciberespacio. El papel de las freenets". En: Antropología del Ciberespacio (pp. 103-119). Abya-Yala, Quito.

Fernández de Rota, J.A. (2009) "El giro etnográfico del patrimonio". En: Fronteras, patrimonio y etnicidad en Iberoamérica (pp. 155-168). Signatura Demos, Sevilla. 
Puigbo, J. Tardio, P, Ortega, H. (2016) Creando e-SPACIOS para la participación popular en el patrimonio: el caso de la comunidad gay en Sitges, perifèria $\mathrm{x}(\mathrm{y})$, Noviembre 2016

revistes.uab.cat/periferia

Gobierno de España, Ministerio de Educación, Cultura y Deporte. (2005). El patrimonio Cultural y los Itinerarios Culturales del Consejo de Europa: nuevas orientaciones. Itinerarios Europeos, Consejo de Europa: Françoise Tondre. Recuperado el 13 de Noviembre de 2016, desde: http://www.mecd.gob.es/cultura-mecd/dms/mecd/cultura-mecd/areascultura/patrimonio/mc/patrimonioeur/itinerarios-culturaleseuropeos/divulgacion/patrimonio itinerarios.pdf

Picciuolo, J.L. (1998) "Dentro y fuera de la pantalla: apuntes para una etnografía del ciberespacio". En Antropología del Ciberespacio (pp. 9-23). Abya-Yala, Quito.

Pizza, G. P. (2005). Antonio Gramsci y la antropología médica contemporánea. Hegemonía, "capacidad de actuar" (agency) y transformaciones de la persona. Revista de Antropología Social, No 14, pp.15-32.

http://dx.doi.org/10.4324/9780203625521

Prats, LL. (2015). Concepto y gestión del patrimonio local. Cuadernos de Antropología Social: Perspectivas contemporáneas en la investigación y la gestión. Universidad de Buenos Aires. No 21, 17-35.

Sarraméa, A. (1999) "Antropología de lo político: sociedad virtual y movilizaciones sociales". En Antropología del Ciberespacio (pp. 88-103). Abya-Yala, Quito.

Hufford. M. (1994) Conserving culture: a new discourse on heritage. American Folklife Center. University of Illinois Press: Chicago. 